\title{
NA-17/MAGE-3.A2/NY-ESO-1 Peptide Vaccine
}

National Cancer Institute

\section{Source}

National Cancer Institute. NA-17/MAGE-3.A2/NY-ESO-1 Peptide Vaccine. NCI Thesaurus. Code C96042.

A peptide cancer vaccine consisting of peptides derived from the melanoma antigen NA17, the human leukocyte antigen HLA-A2-restricted human melanoma antigen 3 (MAGE3.A2) and the cancer-testis antigen (NY-ESO-1), with potential immunostimulating and antineoplastic activities. Upon administration, the NA-17/MAGE-3.A2/NY-ESO-1 peptide vaccine may stimulate the immune system to mount a cytotoxic $T$-cell $(C T L)$ response against tumor cells expressing NA-17, MAGE-3.A2 and NY-ESO-1, resulting in tumor cell lysis. The tumor-associated antigens (TAAs) NA-17, MAGE-3.A2 and NY-ESO-1 are overexpressed in a variety of cancer cell types. 